\title{
Multiple Density Subdural Hematomas
}

\author{
Divye Prakash Tiwari, Vivek Sharma*, Janak Raj \\ Department of Neurosurgery, Institute of Medical Sciences, Banaras Hindu University, Varanasi, India \\ Email: kamlatiwari7@gmail.com, luckin2010@gmail.com, Neurosurgeryimsbhu@gmail.com
}

Received November 10, 2013; revised December 10, 2013; accepted December 17, 2013

Copyright (C) 2014 Divye Prakash Tiwari et al. This is an open access article distributed under the Creative Commons Attribution License, which permits unrestricted use, distribution, and reproduction in any medium, provided the original work is properly cited. In accordance of the Creative Commons Attribution License all Copyrights (C) 2014 are reserved for SCIRP and the owner of the intellectual property Divye Prakash Tiwari et al. All Copyright (C 2014 are guarded by law and by SCIRP as a guardian.

\begin{abstract}
The chronic subdural hematoma is a well known entity in old age and the treatment is very challenging. Treatment protocol depends upon the radiological finding. The loculation and multilayering indicates the bleeding at multiple times and respectively found in $13.2 \%$ and $13.6 \%$, which was more common in patients more than 75 years. Burr hole evacuation was the treatment of choice except in recurrent cases where craniotomy was performed.
\end{abstract}

\section{KEYWORDS}

\section{Chronic Subdural Hematoma; Computed Tomography; Burr Hole; Loculation; Head Injury}

\section{Introduction}

Chronic subdural hematoma (cSDH) has higher incidence among older age persons. Chronic SDH has a variety of imaging characteristics in computed tomography scan: low, intermediate, or high density relative to brain parenchyma. Mixed density subdural hematoma suggests repeated episodes of acute bleeding are due to multiple episodes of trauma. Chronic subdural hematoma is one of the most common diseases treated in neurosurgery with a significant recurrence rate. It may be associated with high morbidity and mortality, depending on the size of the hematoma, the mass effect, and the presence and severity of other associated brain injuries. Computerized tomography (CT) scanning remains the most important diagnostic test for this disorder [1]. Acute trauma on the patients with cSDH may develop acute bleeding over the cSDH, which would produce mixed density [2]. Multiple densities of cSDH may result from multiple episodes of trauma. Aim of this study was to investigate the density and frequency of mixed density chronic subdural hematoma and relationship between the recurrences of cSDH after treatment.

\section{Patients and Methods}

The two hundred twelve patients with diagnosed chronic

"Corresponding author. subdural hematoma from 2008 to 2013 were taken into study. Radiological appearance of all cases was divided into hypodense, isodense, multilayered and loculated. History of head injury was evident in 170 (80.1\%) patients.

\section{Age Distribution}

Maximum numbers of cases [81 (38.2\%)] were found in age group $>75$ years and 35 cases were present in $<55$ years group. Density of chronic subdural hematoma was hypodense (<25 HU) in $48(22.6 \%)$ patients and isodense (25 - 35 HU) in 107 (50.4\%) patients. Radiologically chronic subdural hematoma was found multilayered (Figure 1) in 29 (13.6\%) cases and it was loculated (Figures 2-4) in 28 (13.2\%) patients. The $\mathrm{X}^{2}$ and $\mathrm{p}$ value were 2.52 and 0.98 respectively on applying $\mathrm{X}^{2}$ chi square test (Table 1 ). There was no statistically significant association between the age distribution and radiological finding.

\section{Etiology}

We identified the causes of head trauma in 141 patients (66.5\%). Minor domestic fall in bathroom/stairs was the most common cause in 107 (50.4\%) cases of chronic subdural hematoma. Road traffic accident was found in 34 (16.04\%) patients as the cause of chronic subdural hematoma. The causative agent could not be identified in 


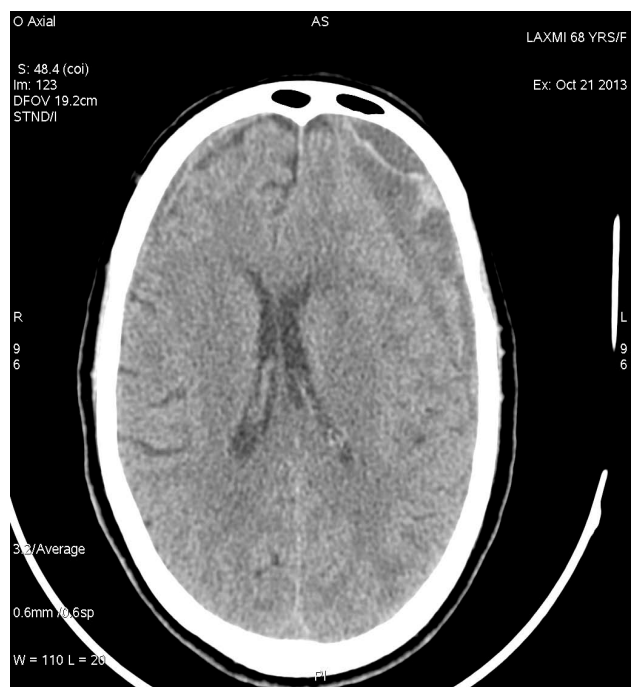

Figure 1. Multilayer chronic subdural hematoma.

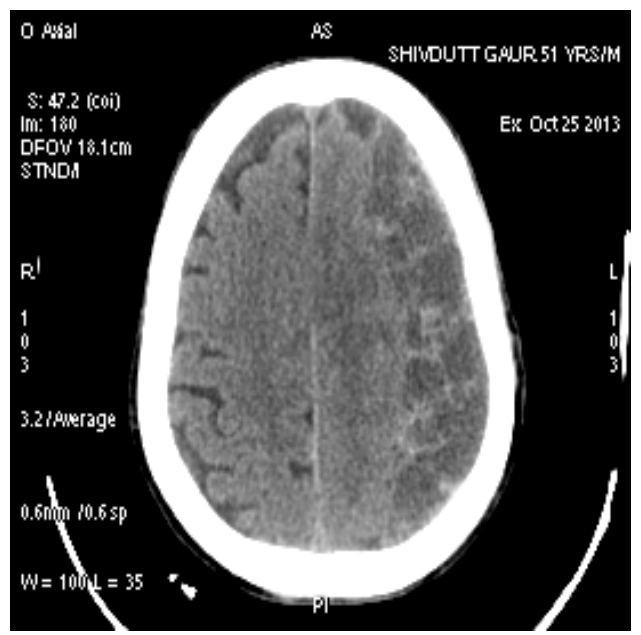

Figure 2. Multilocular chronic subdural hematoma.

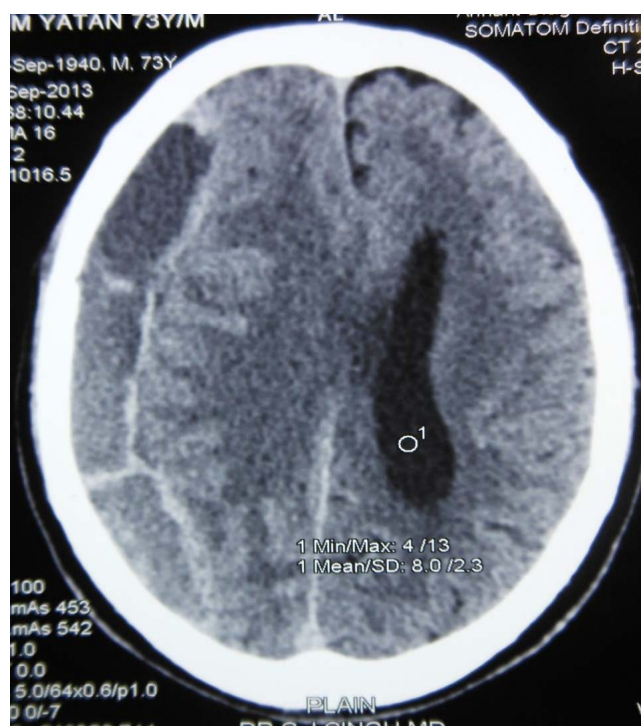

Figure 3. Loculated subdural hematoma.

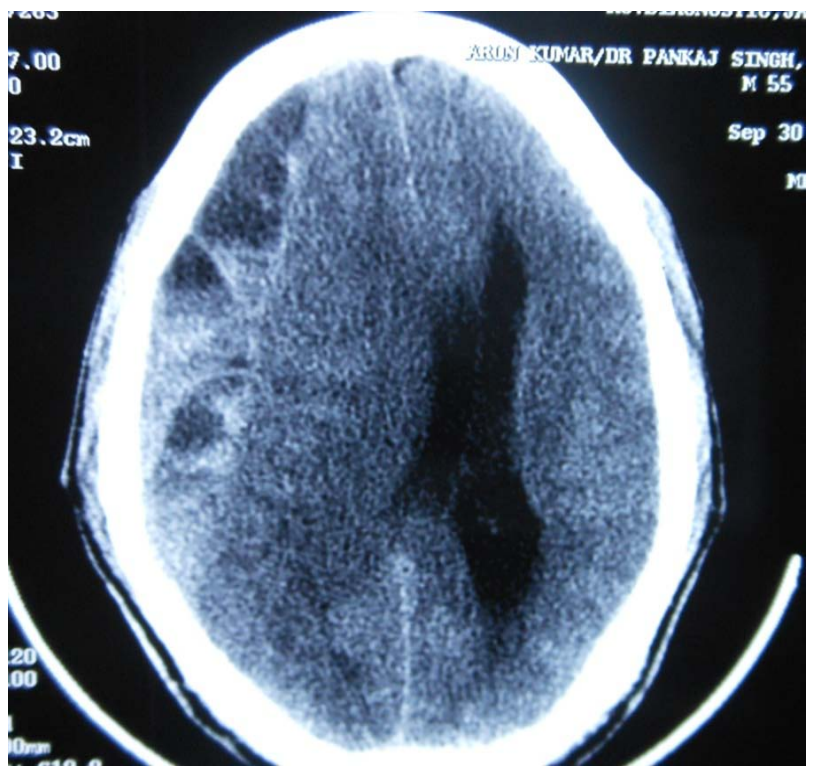

Figure 4. Loculated chronic subdural hematoma.

71 (33.49\%) cases. The cause was identifiable in 16 (55.17\%) multilayered and 14 (50\%) loculated chronic subdural hematoma. The $\mathrm{X}^{2}$ and $\mathrm{p}$ value were 16.75 and 0.01 respectively after applying the Chi square test (Table 2). Thus the radiological findings had significant association with the mode of trauma.

\section{Treatment}

We favored pre/post coronal burr-hole drainage and irrigation under local anesthesia in 169 (79.72\%) patients. The sixteen patients were subjected to craniotomy while the expectant treatment was offered to 27 (12.74\%) cases. We performed craniotomy for 5 patients with multilayered hematomas and in 4 patients with loculated hematomas because the clot was large and was not easily suckable with saline irrigation. After applying the Chi square test, the value of $\mathrm{X}^{2}$ and $\mathrm{p}$ were 14.44 and 0.025 (Table 3). This was statistically significant and there was significant association between radiological picture and type of treatment offered.

\section{Recurrence}

Two patients of hypodense chronic subdural hematoma had recurrence while overall 20 (11.8\%) patients out of 169 treated with burr hole and irrigation developed recurrence in postoperative period. Among patients with multilayered subdural hematoma treated with burr holes and irrigation, six cases had recurrence. One patient each out of 5 and 4 of multilayered and loculated cSDH treated with craniotomy developed recurrence. The $\mathrm{X}^{2}$ and $\mathrm{p}$ value were 28.84 and 0.001 after applying Chi square test which was statistically significant in burr hole group (Table 4). It could not be applied for recurrence in other 
Table 1. CT picture of subdural hematoma with age of patient.

\begin{tabular}{|c|c|c|c|c|c|}
\hline Radiological feature & Age $<55$ & Age 56 - 65 & Age 66 - 75 & Age $>75$ & Irrespective of age \\
\hline Hypodense & $8(16.67 \%)$ & $09(18.75 \%)$ & $12(25.00 \%)$ & $19(39.58 \%)$ & $48(22.64 \%)$ \\
\hline Isodense & 17 (15.89\%) & 21 (19.63\%) & 27 (25.23\%) & 42 (39.25\%) & 107 (50.54\%) \\
\hline Multilayered & $4(13.79 \%)$ & 6 (20.69\%) & $8(27.59 \%)$ & $11(37.97 \%)$ & $29(13.68 \%)$ \\
\hline Loculated & $6(21.43 \%)$ & 8 (28.57\%) & $5(17.86 \%)$ & 9 (32.14\%) & 28 (13.21\%) \\
\hline Total & 35 (16.51\%) & $44(20.75 \%)$ & 52 (24.53\%) & $81(38.21 \%)$ & 212 (100.00\%) \\
\hline
\end{tabular}

Table 2. Etiology versus radiological feature in chronic subdural hematoma.

\begin{tabular}{cccc}
\hline Radiological feature & Road Traffic Accident & Trivial Trauma & Obscure \\
\hline Hypodense & $4(8.33 \%)$ & $32(66.67 \%)$ & $12(25.00 \%)$ \\
Isodense & $17(15.89 \%)$ & $58(54.21 \%)$ & $32(29.91 \%)$ \\
Multilayered & $8(27.59 \%)$ & $8(27.59 \%)$ & $13(44.83 \%)$ \\
Loculated & $5(17.86 \%)$ & $9(32.84 \%)$ & $14(50.00 \%)$ \\
Total & $34(16.04 \%)$ & $107(50.47 \%)$ & $71(33.49 \%)$ \\
Chi Square test & $\mathrm{X}^{2}=16.75$ & $\mathrm{df}=6$ & $\mathrm{P}=0.01$ \\
\hline
\end{tabular}

Table 3. Surgical procedure in respect of radiological feature.

\begin{tabular}{|c|c|c|c|c|}
\hline Radiological feature & Burr holes & Craniotomy & Expectant treatment & Irrespective of operative option \\
\hline Hypodense & $34(78.83 \%)$ & $4(8.33 \%)$ & $10(22.83 \%)$ & $48(22.64 \%)$ \\
\hline Isodense & $90(84.11 \%)$ & $3(2.80 \%)$ & $14(13.08 \%)$ & $107(50.47 \%)$ \\
\hline Multilayered & 22 (75.83\%) & 5 (17.24\%) & $2(6.90 \%)$ & 29 (13.68\%) \\
\hline Loculated & $23(82.14 \%)$ & $4(14.29 \%)$ & $1(3.57 \%)$ & $28(13.21 \%)$ \\
\hline Total & 169 (79.72\%) & $16(7.55 \%)$ & 27 (12.74\%) & 212 (100.00\%) \\
\hline Chi square test & \multicolumn{4}{|c|}{$\mathrm{df}=6, \mathrm{X}^{2}=14.44, \mathrm{p}=0.025$} \\
\hline
\end{tabular}

Table 4. Recurrence in different mode of treatment in various radiological groups.

\begin{tabular}{|c|c|c|c|c|c|c|}
\hline \multirow{2}{*}{ Radiological feature } & \multicolumn{2}{|c|}{ Recurrence in Burr holes study group } & \multicolumn{2}{|c|}{ Recurrence in craniotomy study group } & \multicolumn{2}{|c|}{$\begin{array}{l}\text { Recurrence in Expectant } \\
\text { treatment study group }\end{array}$} \\
\hline & No & Yes & No & Yes & No & Yes \\
\hline Hypodense & $32(94.12 \%)$ & $02(5.88 \%)$ & $4(100.0 \%)$ & $0(0)$ & $9(90.00 \%)$ & $1(10.6 \%)$ \\
\hline Isodense & 87 (96.67\%) & $3(3.33 \%)$ & $3(100.0 \%)$ & $0(0)$ & $13(92.86 \%)$ & $1(7.14 \%)$ \\
\hline Multilayered & $16(72.73 \%)$ & $6(27.27 \%)$ & $4(80 \%)$ & $1(20 \%)$ & $1(50 \%)$ & $1(50 \%)$ \\
\hline Chi square test & \multicolumn{2}{|c|}{$\mathrm{X}^{2}=28.84, \mathrm{df}=3, \mathrm{p}<0.001$} & \multicolumn{4}{|c|}{ Test cannot be applied due small sample } \\
\hline
\end{tabular}

treatment modality as number of cases was few.

\section{Discussion}

Chronic subdural hematoma is an encapsulated collection of old blood, mostly or totally liquidified and located between dura mater and arachnoid. Virchow first described it in 1857 as "pachymeningitis hemorrhagica interna". Later Trotter put forward the theory of trauma to the bridging veins as a cause of what he named "subdural hemorrhagic cyst". It has long been recognized that the elderly are more likely to develop subdural hematoma, par- ticularly from minor trauma. The initial trauma to the bridging veins results in hemorrhage in to the subdural space. A day after the hemorrhage, the outer surface of the hematoma is covered by a thin layer of fibrin and fibroblasts. Migration and proliferation of the fibroblasts leads to formation of a membrane over the clot by the fourth day. The outer membrane progressively enlarges and the fibroblasts invade the hematoma and form a thin membrane during the next two weeks [3]. Repeated trauma may cause recurrent bleeding, which would make a lump or a layer of hyper density within hypo or isodense hematoma. Like the repeated micro hemorrhages 
from the outer membrane, repeated trauma may cause acute bleeding over the cSDH as a mechanism of multidensity. The hypodensity was the most common type in CT scanning but there was loculation and multi-layering. Treatment of cSDH is by surgical evacuation, although small hematomas may resolve spontaneously. Patients treated conservatively should be carefully monitored and the scan should be repeated if there is a clinical deterioration [3]. Evacuation through single burrhole was not successful to clear all pockets therefore either multiple burrholes or craniotomy was the treatment of choice in this situation. Burr hole evacuation was found statistically significant.

Increased size of hematoma is attributed to brain atrophy associated with ageing, which may provide the cSDH a potential space to grow [4]. There was significant association between age and radiological finding. Furthermore, it has been speculated that left over pockets of subdural hematoma may lead to inappropriate brain re-expansion postoperatively and thereby give the potential space for re-accumulation of the hematoma [5].

A layered type of the hematoma density may result from prolonged recumbence, which separates the blood components and fluid [6]. The CT density coefficients reflect the blood components. If the patient maintains lying position for a long time, heavier components sink by the gravity.

Theoretically, the mixed density results from three hypotheses. The first situation is acute bleeding; the difference between solid clot and liquid blood may produce the mixed density. The second situation is in sub acute SDHs; resolving hematoma may appear peripheral hypodensity with central hyper density during transitional period. The reason is the local difference between the hemolytic activities. Fibrinolysis in CSF is activated from the outside to the inside in order after trauma. The change into the hypodensity is faster in the peripheral region than in the center, where the density of hematoma remains hyper dense for a long time. Mixed or layered types were more common in the oldest age, while isodense or hypodense SDHs were more common the age of less than 70 years. This uneven distribution results from not only the fact that the reserving capacity is maximal in the oldest age, but also the oldest patients are vulnerable to repeated trauma. Also, elderly patients cannot remember their trivial trauma events because of cognitive impairment. The CT density can appear to be mixed density during this transitional period [7]. Multi Burr-hole drainage is sufficient for most patients of multidesity cSDH [8]. Craniotomy may be necessary for those instances in which the subdural collection reaccumulates, the brain fails to expand, or there is some solid hemato- ma [9]. $X^{2}$ and $p$ value were statistically significant in recurrence among burrhole operative group.

\section{Conclusion}

The Mixed density of cSDH is unusual radiological variant and implies to multiple hemorrhages at different stages of time. Draining of one pocket and leaving others may not give neurological improvement. The right strategy of treatment is to evacuate all pockets either through multiple burrrholes or craniotomy. Thus its management is challenging and different from usual cSDH.

Conflict of Interest: Nil.

Funding source: Nil.

\section{REFERENCES}

[1] M. Gelabert-González, M. Iglesias-Pais, A. García-Allut and R. Martínez-Rumbo, "Chronic Subdural Hematoma: Surgical Treatment and Outcome in 1000 Cases," Clinical Neurology Neurosurgery, Vol. 107, No. 3, 2005, pp. 223229. http://dx.doi.org/10.1016/j.clineuro.2004.09.015

[2] K. S. Lee, J. J. Shim, S. M. Yoon, J. W. Doh, I. G. Yun and H. G. Bae, "Acute-on-Chronic Subdural Hematoma: Not Uncommon Events," Journal of Korean Neurosurgery Society, Vol. 50, No. 6, 2011, pp. 512-516. http://dx.doi.org/10.3340/jkns.2011.50.6.512

[3] V. Adhiyaman, M. Asghar, K. N. Ganeshram and B. K. Bhowmick, "Chronic Subdural Hematoma in the Elderly," Postgraduate Medical Journal, Vol. 78, No. 916, 2002, pp. 71-75. http://dx.doi.org/10.1136/pmj.78.916.71

[4] A. Spallone, R. Giuffre, F. M. Gagliardi and R. Vagnozzi, "Chronic Subdural Hematoma in Extremely Aged Patients," European Journal of Neuroscience, Vol. 29, No. 1, 1989, pp. 18-22. http://dx.doi.org/10.1159/000116370

[5] K. Mori and M. Maeda, "Surgical Treatment of Chronic Subdural Hematoma in 500 Consecutive Cases: Clinical Characteristics, Surgical Outcome, Complications, and Recurrence Rate,” Neurologia Medico-Chirurgica (Tokyo), Vol. 41, No. 8, 2001, pp. 371-381. http://dx.doi.org/10.2176/nmc.41.371

[6] K. S. Lee, "Natural History of Chronic Subdural Hematoma," Brain Injury, Vol. 18, No. 4, 2004, pp. 351-358. http://dx.doi.org/10.1080/02699050310001645801

[7] H.-R. Park, K.-S. Lee, J.-J. Shim, et al., "Multiple Densities of the Chronic Subdural Hematoma in CT Scans," Journal of Korean Neurosurgery Society, Vol. 54, No. 1, 2013, pp. 38-41.

[8] J. Y. Lee, H. Ebel, R. I. Ernestus and N. Klug, "Various Surgical Treatments of Chronic Subdural Hematoma and Outcome in 172 Patients: Is Membranectomy Necessary?” Surgical Neurology, Vol. 61, No. 6, 2004, pp. 523-527.

[9] T. M. Markwalder, "Chronic Subdural Hematomas: A Review,” Journal of Neurosurgery, Vol. 54, No. 5, 1981, pp. 637-645. http://dx.doi.org/10.3171/jns.1981.54.5.0637 